\title{
Instability of the pelvic ring and injury severity can be predictors of death in patients with pelvic ring fractures: a retrospective study
}

\author{
Toshiya Tachibana $\cdot$ Hideyuki Yokoi $\cdot$ \\ Manabu Kirita $\cdot$ Seishiro Marukawa \\ Shinichi Yoshiya
}

Received: 20 August 2008/Accepted: 13 March 2009/Published online: 2 April 2009

(C) Springer-Verlag 2009

\begin{abstract}
Background The correlation between fracture type and mortality in patients with pelvic fracture has been previously investigated. The purpose of this study was to determine whether instability of the pelvic ring as assessed by Tile's classification is a predictor of death in patients with pelvic fractures.

Materials and methods The clinical course of consecutive patients with pelvic fractures was retrospectively reviewed. Eighty-seven patients with pelvic ring fractures were included in the study. As potential predictive factors, fracture type according to Tile's classification, and generally used traumatic parameters (injury severity score, revised trauma score, and probability of survival) were analyzed.

Results The mortality was significantly higher in patients with unstable fracture patterns $(P<0.05)$. In non-survivors, index values of traumatic parameters were more severe than those in survivors $(P<0.05)$.

Conclusion The present study suggests that patients with unstable pelvic fractures have an increased risk of death associated with exacerbated injury severity.
\end{abstract}

Keywords Pelvic ring fracture $\cdot$ Mortality $\cdot$ Instability · Injury severity

T. Tachibana $(\bowtie) \cdot$ H. Yokoi $\cdot$ S. Yoshiya

Department of Orthopaedic Surgery, Hyogo College

of Medicine, Nishinomiya, Hyogo 663-8501, Japan

e-mail: tachi@hyo-med.ac.jp

M. Kirita $\cdot$ S. Marukawa

Department of Emergency and Critical Care Medicine, Hyogo

College of Medicine, Nishinomiya, Hyogo 663-8501, Japan

\section{Introduction}

Pelvic fracture is one of the most challenging fractures for orthopaedic and trauma surgeons to treat because of the high mortality and high frequency of the occurrence of associated injuries. In the assessment of patients with pelvic fractures, instability of the pelvis ring is thought to be an important factor affecting the treatment decision. For unstable pelvic fractures, use of clamps and external fixators are considered to stabilize the pelvic ring so reducing bleeding.

The correlation between fracture type and mortality in patients with pelvic fracture has been previously investigated. However, the fracture classification system adopted in these analyses has varied from study to study, and thus the prognosis of patients with this injury remains difficult to predict. Since pelvic ring instability is the key to determining injury severity, we hypothesized that stabilitybased Tile's fracture type classification might be a useful predictor of patient mortality [1]. To test this hypothesis, we assessed the relationship between mortality and fracture stability using Tile's classification together with an examination of injury severity of each patient.

\section{Materials and methods}

A retrospective study of pelvic fractures was performed for consecutive patients admitted to the emergency and critical care units at our institution between April 2000 and June 2004. Of 136 patients with pelvic fractures, 92 were diagnosed with pelvic ring fractures, and the remaining 44 with acetabular fractures. Of 92 patients with pelvic ring fractures, 5 patients with cardio pulmonary arrest on arrival were excluded, leaving 87 patients as the study population. 
Fifty-seven patients sustained injuries in road traffic collisions, 24 patients were injured as a results of falls, and the remaining 6 patients were involved in other accidents.

After initial evaluation and resuscitation, all patients underwent plain radiography of the pelvis and sonography in the emergency room followed by the focused assessment with sonography for trauma (FAST) protocol. For detailed fracture assessment, all patients underwent plane computed tomographic (CT) scanning of the pelvis. When the presence of substantial hematoma was identified, enhanced CT and angiography were additionally performed after stabilizing the hemodynamics of the patient by fluid. Patients who had angiographic evidence of extravasation underwent transcatheter arterial embolization (TAE). The fractures deemed to be unstable were fixed immediately by external fixators in the operating room.

For each patient, the fracture type was classified by univariate analysis using the Tile's classification, which divides pelvic ring fractures into types $\mathrm{A}, \mathrm{B}$, and $\mathrm{C}$ based on the degree of pelvic instability; type A is stable, type B has rotational instability, and type $\mathrm{C}$ has rotational and vertical instability. All patients underwent CT scanning, and fracture type was categorized using plain radiographs and CT scan images. Especially, vertical instability of the fracture was assessed by an outlet radiograph and threedimensionally reconstructed CT images. Then, patient mortality was compared between the groups. As additional indicators of injury severity, injury severity score (ISS: an anatomic parameter), revised trauma score (RTS: a physiologic parameter) and probability of survival (Ps) were also evaluated as predictive factors. These parameters were recorded by physicians immediately after admission of the patients, and assessment and scoring for each patient were performed correctly by one senior physician (MK).

In the statistical analysis, $\chi^{2}$ analysis was performed with Excel (Microsoft, Redmond, WA, USA), analysis of variant was performed using StatView (SAS Institute, Cary, NC, USA), and Logistic regression analysis was performed using SPSS (SPSS, Chicago, IL, USA). A $P$ value of $<0.05$ was considered to indicate significance.

The study conforms to the Declaration of Helsinki and was approved by the Istitutional Review Board/Ethical Committee. All the enrolled patients provided informed consent.

\section{Results}

Among the 87 patients included in the study, 70 survived and 17 died during the subsequent treatment at our department. There was no significant difference in sex and age between survivors and non-survivors (Table 1). The mortality rate in each group with different fracture type
Table 1 Comparison of fracture patterns and trauma scores between survivors and non-survivors

\begin{tabular}{lll}
\hline & Survivors & Non-survivors \\
\hline Sex & & \\
Male & 46 & 8 \\
Female & 24 & 9 \\
Age & $39.8 \pm 19.1$ & $50.3 \pm 23.1$ \\
Tile's & & \\
A & 36 & $3^{*}$ \\
B & 30 & $10^{*}$ \\
C & 4 & $4^{*}$ \\
ISS & $20.0 \pm 8.6$ & $37.5 \pm 8.8^{*}$ \\
RTS & $7.1 \pm 0.9$ & $3.4 \pm 1.9^{*}$ \\
Ps & $0.88 \pm 0.14$ & $0.30 \pm 0.25^{*}$ \\
\hline
\end{tabular}

ISS injury severity score, $R T S$ revised trauma score, $P$ s probability of survival

$* P<0.05$

Table 2 Predictive factors of death

\begin{tabular}{lllll}
\hline \multirow{5}{*}{} & \multicolumn{2}{l}{ Logistic regression analysis } \\
\cline { 3 - 5 } & $P$ value & Odds ratio & $95 \%$ CI & \\
\cline { 3 - 5 } & & & Lower & Upper \\
\hline Tile's & $0.041^{*}$ & 6.841 & 1.086 & 43.073 \\
Sex & 0.592 & & & \\
Age & 0.119 & & & \\
ISS & $0.033^{*}$ & 1.213 & 1.015 & 1.449 \\
RTS & $0.022^{*}$ & 0.173 & 0.039 & 0.775 \\
Ps & 0.134 & & & \\
\hline
\end{tabular}

$I S S$ injury severity score, $R T S$ revised trauma score, $P S$ probability of survival, $C I$ confidence interval

$* P<0.05$

was $3 / 39$ (7.7\%), 10/40 (25\%), and 4/8 (50\%) in types A, $\mathrm{B}$, and $\mathrm{C}$, respectively (Table 1 ). Thus, the mortality rate was significantly higher in patients with unstable fracture types B and C $(P<0.05$, Table 1$)$. ISS, RTS, and Ps were significantly more severe in non-survivors indicating a close relationship between injury severity and mortality (Table 1). Logistic regression analysis for potentially influencing factors on mortality demonstrated that Tile's classification, ISS, and RTS significantly affect the survival rate (Table 2).

\section{Discussion}

The mortality of patients with pelvic ring fractures has been previously described in the literature [2-16]. As potential predictors of mortality, several factors including 
fracture type [2-12] injury severity [2, 3, 6, 7, 12, 13] and hemo-dynamics $[14,15]$ have been evaluated. Among those factors, the possible correlation between fracture type and mortality has remained controversial. One of the variables that has been most criticized in past studies is the system for classifying the fracture types.

Eastridge [2] compared the prognosis of patients with stable pelvic ring fracture patterns and patients with unstable fractures patterns using Young-Burgess's classification which divides fracture patterns based on the energy direction. They analyzed patients with stable fracture patterns who required on-going resuscitation and who underwent celiotomy and/or angiography, and showed that abdominal hemorrhage was responsible for hypotension in $85 \%$, while $25 \%$ of the patients died. However, in patients with unstable fracture patterns, hemorrhage was predominantly from a pelvic source, as shown by positive angiograms in 59\%; $52 \%$ of the patients died. Thus, they concluded that in patients with unstable fractures patterns, even in the presence of hemoperitoneum, consideration should be given to angiography before celiotomy. They also showed that patients with unstable fracture patterns had a higher mortality rate than patients with stable fracture patterns. This has been shown in other studies, adopting Tile's classification. Shindo et al. [8] reported that patients with Tile's type $\mathrm{C}$ fractures had a significantly higher mortality rate than those with stable fracture patterns of type A. In contrast, Hagiwara et al. [3] suggested that mortality did not correlate with fracture type when TAE was performed. However, they failed to compare the mortality rate between patients with and without TEA. In Key and Conwell's classification, pelvis stability was not considered and their study examining the relationship between mortality and this fracture type failed to show any significant relationship [7]. No statistical analysis was presented in other studies, thus the results cannot be considered conclusive $[4,5,9-11]$. The present study showed a significant correlation between mortality and fracture type based on Tile's classification system which is based on pelvic stability. Therefore stability of the pelvic ring as assessed by this system can be a useful indicator in predicting mortality of patient with pelvic ring fractures.

In the relationship between patient mortality and overall injury severity, Poole [6] showed that ISS was more severe in patients with unstable pelvic fractures than in those with stable fractures, and also more severe in non-survivors than survivors, indicating a close relationship between injury severity and mortality. Parreira [7] reported that the outcome of patients with pelvic fractures due to blunt trauma correlated with the severity of associated injuries and physiological derangement on admission. Lunsjo [12] reported that ISS is the most important predictor in defining mortality in patients with pelvic fractures. In the present study, all traumatic parameters (ISS, RTS, and Ps) were more severe in non-survivors. Moreover, logistic regression analysis showed that ISS and RTS could be used as predictive factors of death in patients with pelvic fractures.

In the present study, both instability of the pelvic ring and injury severity were correlated with mortality of patients with pelvic ring fractures. Thus, all parameters adopted in the study (Tile's classification, ISS, RTS) could be regarded as potent predictive factors for death of the patients. Assessment of the patient condition in various aspects is thought to be critical in the initial clinical evaluation.

In conclusion, Tile's classification significantly correlates with mortality, and thus is a useful classification system for management of patients with pelvic fractures. Moreover, a close relationship between fracture stability as assessed by this system and injury severity was also suggested. In the treatment of patients with pelvic fractures, surgeons should evaluate fracture stability and injury severity immediately after admission to predict the prognosis and establish the initial treatment plan.

Acknowledgment We thank Ms. Janina Tubby for her help in editing the manuscript.

Conflict of interest statement The authors declare that they have no competing interests.

\section{References}

1. Tile M (1995) Classification of fractures of the pelvis and acetabulum. In: Tile M (ed) Fructures of the pelvis and acetabulum, 2nd edn. Williams \& Wilkins, London, pp 66-101

2. Eastridge BJ, Starr A, Minei JP, O'Keefe GE (2002) The importance of fracture pattern in guiding therapeutic decisionmaking in patients with hemorrhagic shock and pelvic ring disruptions. J Trauma 53:446-451

3. Hagiwara A, Minakawa K, Fukushima H, Murata A, Masuda H, Shimazaki S (2003) Predictors of death in patients with lifethreatening pelvic hemorrhage after successful transcatheter arterial emblization. J Trauma 55:696-703

4. Gänsslen A, Pholemann T, Lobenhoffer Ph, Tscherne H (1996) Epidemiology of pelvic ring injuries. Injury 27(1):A13-A20

5. Chong KH, DeCoster T, Osler T, Robinson B (1997) Pelvic fractures and mortality. Iowa Orthop J 17:110-114

6. Poole GV, Ward F, Muakkassa FF, Hsu HSH, Griswold JA, Rhodes RB (1991) Pelvic fracture form major blunt trauma. Outcome is determined by associated injuries. Ann Surg 231:532-538

7. Parreira JG, Coimbra R, Rasslan S, Oliveira A, Fregonez M, Mercadante M (2000) The role of associated injuries on outcome of blunt trauma patients sustaining pelvic fractures. Injury 31:677-682

8. Shindo M, Tanaka K, Agira T, Wakita R, Nishimaki H, Souma K (2001) Pelvic fractures in vehicular trauma patients. J Japn Council Traffic Sci 1:23-28

9. Dalal SA, Burgess AR, Siegel JH, Young JW, Brumback RJ, Poka A, Dunham M, Gens D, Bathon H (1989) Pelvic fracture in multiple trauma: classification by mechanism is key to pattern of 
organ injury, resuscitative requirements and outcom. J Trauma 29:981-1002

10. Rothenberger DA, Fischer RP, Strate RG, Velasco R, Perry JF (1978) The mortality associated with pelvic fractures. Surgery 84:356-362

11. Looser KG, Combie HD (1976) Pelvic fractures: an anatomical guide to severity of injury. Am J Surg 132:638-642

12. Lunsjo K, Tadros A, Hauggaard A, Blomgren R, Kopke J, AbuZidan FM (2008) Assosiated injuries and not fracture instability predict mortality in pelvic fractures: a prospective study of 100 patients. J Trauma 62:687-691
13. Wubben RC (1996) Mortality rate of pelvic fracture patients. Wisconsin Med J 95:702-704

14. Mucha P, Farnell MB (1984) Analysis of pelvic fracture management. J Trauma 24:379-386

15. Allen CF, Goslar PW, Barry M, Christiansen T (2000) Management guidelines for hypotensive pelvic fracture patients. Am Surg 66:735-738

16. Moreno C, Moore EE, Rosenberger A, Cleveland HC (1986) Hemorrhage associated with major pelvic fracture: a multispecialty challenge. J Trauma 26:987-994 